\title{
Periodic Boundary Value Problems for a Class of Impulsive Functional Differential Equations of Hybrid Type
}

\author{
Guoping Chen \\ Department of Mathematics, Jishou University, Jishou, Hunan 416000, China \\ Correspondence should be addressed to Guoping Chen; cgp_pgc@163.com
}

Received 16 October 2013; Accepted 13 November 2013; Published 4 February 2014

Academic Editors: T. Y. Kam, M. Mei, and X.-S. Yang

Copyright ( 2014 Guoping Chen. This is an open access article distributed under the Creative Commons Attribution License, which permits unrestricted use, distribution, and reproduction in any medium, provided the original work is properly cited.

\begin{abstract}
This paper is concerned with the existence of extreme solutions of periodic boundary value problems for a class of first-order impulsive functional differential equations of hybrid type. We obtain the sufficient conditions for existence of extreme solutions by using upper and lower solutions method coupled with monotone iterative technique.
\end{abstract}

\section{Introduction}

The theory of impulsive differential equations is now being recognized to be not only richer than the corresponding theory of differential equations without impulses but also represents a more natural framework for mathematical modeling of many real-world phenomena [1-3]. Significant progress has been made in the theory of systems of impulsive differential equations in recent years (see [4-18] and the references cited therein). It is well known that the monotone iterative technique offers an approach for obtaining approximate solutions of nonlinear differential equations; for details, see [19] and the references therein. There also exist several works devoted to the applications of this technique to periodic boundary value problems of impulsive differential equations; see $[20-26]$. In $[27,28]$, the authors introduce a new concept of upper and lower solutions for periodic boundary value problems of a class of first-order functional differential equations. In paper [23], the authors applied this new concept to study the periodic boundary value problems for first-order impulsive functional differential equations. Motivated by [23, 27,28 ], we will study periodic boundary value problem for the first-order impulsive functional differential equation of hybrid type

$$
\begin{array}{r}
x^{\prime}(t)=f(t, x(t), x(\theta(t)),(K x)(t),(H x)(t)), \\
t \neq t_{k}, \quad t \in J=[0, T], \quad T>0,
\end{array}
$$

$$
\begin{gathered}
\Delta x\left(t_{k}\right)=I_{k}\left(x\left(t_{k}\right)\right), \quad k=1,2, \ldots, m, \\
x(0)=x(T),
\end{gathered}
$$

where $f \in C\left(J \times R^{4}, R\right), \theta \in C(J, J), I_{k} \in C(R, R), 0=t_{0}<$ $t_{1}<t_{2}<\cdots<t_{m}<t_{m+1}=T, \Delta x\left(t_{k}\right)=x\left(t_{k}^{+}\right)-x\left(t_{k}^{-}\right)$ denotes the jump of $x(t)$ at $t=t_{k}, x\left(t_{k}^{+}\right)$, and $x\left(t_{k}^{-}\right)$represent the right and left limits of $x(t)$ at $t=t_{k}$, respectively. Denote $J^{\prime}=J \backslash\left\{t_{1}, t_{2}, \ldots, t_{m}\right\}$. The integral part in (1) is defined by

$$
\begin{aligned}
& (K x)(t)=\int_{0}^{\gamma(t)} k(t, s) x(\delta(s)) d s, \\
& (H x)(t)=\int_{0}^{T} h(t, s) x(\sigma(s)) d s,
\end{aligned}
$$

where $\gamma, \delta, \sigma \in C(J, J), k \in C\left(D, R^{+}\right), D=\{(t, s) \in J \times J$ : $0 \leq s \leq \gamma(t) \leq T\}, h \in C\left(J \times J, R^{+}\right), R^{+}=[0, \infty), K_{0}=$ $\max \{k(t, s):(t, s) \in D\}$, and $H_{0}=\max \{h(t, s):(t, s) \in J \times J\}$.

Let $P C(J)=\left\{u: J \rightarrow R, u\right.$ is continuous for $t \in J, t \neq t_{k}$, $u\left(t_{i}^{+}\right)$, and $u\left(t_{i}^{-}\right)$exist, and $\left.u\left(t_{i}^{-}\right)=u\left(t_{i}\right), i=1,2, \ldots, m\right\}$. $P C^{1}(J)=\{u \in P C(J): u$ is continuously differentiable for 
$\left.t \in J, t \neq t_{k}\right\} . P C(J)$ and $P C^{1}(J)$ are Banach spaces with the norms

$$
\begin{gathered}
\|u\|_{P C(J)}=\sup \{|u(t)|: t \in J\}, \\
\|u\|_{P C^{1}(J)}=\max \left\{\|u\|_{P C(J)},\left\|u^{\prime}\right\|_{P C(J)}\right\} .
\end{gathered}
$$

By a solution of (1), we mean a $u \in P C^{1}(J)$ for which problem (1) is satisfied.

Note that (1) has a very general form; as special instances resulting from (1), one can have impulsive differential equations with deviating arguments and impulsive differential equations with the Volterra or Fredholm operators. For example, if $f$ does not include $K x$ and $H x$, then (1) reduces to

$$
\begin{gathered}
x^{\prime}(t)=f(t, x(t), x(\theta(t))), \\
t \neq t_{k}, \quad t \in J=[0, T], \quad T>0, \\
\Delta x\left(t_{k}\right)=I_{k}\left(x\left(t_{k}\right)\right), \quad k=1,2, \ldots, m, \\
x(0)=x(T),
\end{gathered}
$$

periodic boundary problem for impulsive differential equations with deviating arguments, which is discussed in $[22,23]$; when $\theta(t)=\gamma(t)=\delta(t)=\sigma(t) \equiv t$, (1) is the following periodic boundary problem for impulsive integrodifferential equations of mixed type:

$$
\begin{gathered}
x^{\prime}(t)=f(t, x(t),(K x)(t),(H x)(t)), \\
t \neq t_{k}, \quad t \in J=[0, T], \quad T>0, \\
\Delta x\left(t_{k}\right)=I_{k}\left(x\left(t_{k}\right)\right), \quad k=1,2, \ldots, m, \\
x(0)=x(T) ;
\end{gathered}
$$

similar problems are also discussed in [24-26].

\section{Preliminaries}

To apply the method of upper and lower solutions, we need the concept of lower solution and upper solution for (1).

Definition 1. A function $\alpha \in P C^{1}(J)$ is a lower solution of (1) if there exist $M_{1}>0, M_{i} \geq 0, i=2,3,4$, and $0 \leq L_{k}<1$, $k=1,2, \ldots, m$, such that

$$
\begin{array}{r}
\alpha^{\prime}(t) \leq f(t, \alpha(t), \alpha(\theta(t)),(K \alpha)(t),(H \alpha)(t))-a_{\alpha}(t), \\
t \neq t_{k}, \quad t \in J, \\
\Delta \alpha\left(t_{k}\right) \leq I_{k}\left(\alpha\left(t_{k}\right)\right)-L_{k} a_{\alpha k}, \quad k=1,2, \ldots, m ;
\end{array}
$$

similarly, a function $\beta \in P C^{1}(J)$ is an upper solution of (1) if

$$
\begin{gathered}
\beta^{\prime}(t) \geq f(t, \beta(t), \beta(\theta(t)),(K \beta)(t),(H \beta)(t))+a_{\beta}(t), \\
t \neq t_{k}, \quad t \in J, \\
\Delta \beta\left(t_{k}\right) \geq I_{k}\left(\beta\left(t_{k}\right)\right)+L_{k} a_{\beta k}, \quad k=1,2, \ldots, m,
\end{gathered}
$$

where

$$
\begin{aligned}
& a_{\alpha}(t)= \begin{cases}0, & \text { if } \alpha(0) \leq \alpha(T), \\
\left(M_{1} t+M_{2} \theta(t)\right. & \\
+M_{3} K_{0} \int_{0}^{\gamma(t)} \delta(s) d s & \\
\left.+M_{4} H_{0} \int_{0}^{T} \sigma(s) d s+1\right) & \\
\times(T)^{-1}[\alpha(0)-\alpha(T)], & \text { if } \alpha(0)>\alpha(T),\end{cases} \\
& a_{\alpha k}= \begin{cases}0, & \text { if } \alpha(0) \leq \alpha(T), \\
\frac{t_{k}}{T}[\alpha(0)-\alpha(T)], & \text { if } \alpha(0)>\alpha(T),\end{cases}
\end{aligned}
$$

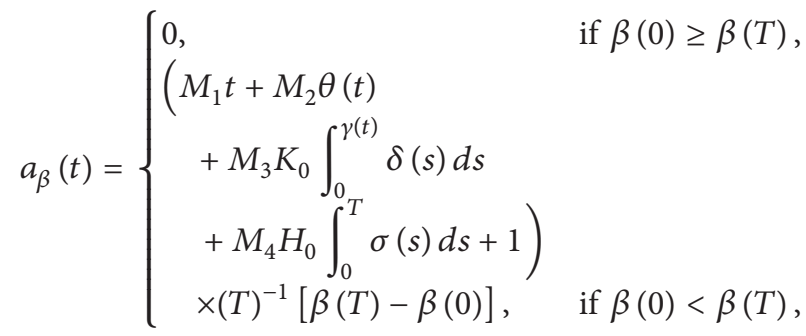

$$
\begin{aligned}
& a_{\beta k}= \begin{cases}0, & \text { if } \beta(0) \geq \beta(T), \\
\frac{t_{k}}{T}[\beta(T)-\beta(0)], & \text { if } \beta(0)<\beta(T) .\end{cases}
\end{aligned}
$$

In what follows, we define the set

$$
[u, v]=\{w \in P C(J): u(t) \leq w(t) \leq v(t), t \in J\}
$$

for $u, v \in P C(J)$ and $u(t) \leq v(t), t \in J$.

For the sake of convenience, we list the following conditions.

$\left(\mathrm{H}_{1}\right)$ Assume that $\alpha(t) \leq \beta(t)$ for $t \in J$, and there exist $M_{1}>0$ and $M_{i} \geq 0, i=2,3,4$, such that

$$
\begin{array}{r}
f\left(t, x_{1}, x_{2}, x_{3}, x_{4}\right)-f\left(t, y_{1}, y_{2}, y_{3}, y_{4}\right) \\
\geq-M_{1}\left(x_{1}-y_{1}\right)-M_{2}\left(x_{2}-y_{2}\right) \\
\quad-M_{3}\left(x_{3}-y_{3}\right)-M_{4}\left(x_{4}-y_{4}\right),
\end{array}
$$

for $\alpha(t) \leq y_{1} \leq x_{1} \leq \beta(t), \alpha(\theta(t)) \leq y_{2} \leq x_{2} \leq$ $\beta(\theta(t)),(K \alpha)(t) \leq y_{3} \leq x_{3} \leq(K \beta)(t)$, and $(H \alpha)(t) \leq$ $y_{4} \leq x_{4} \leq(H \beta)(t), t \in J$.

$\left(\mathrm{H}_{2}\right)$ There exists $L_{k}$ with $0 \leq L_{k}<1, k=1,2, \ldots, m$, such that

$$
\begin{aligned}
& I_{k}(x)-I_{k}(u) \geq-L_{k}(x-u), \\
& \text { for } \alpha\left(t_{k}\right) \leq u\left(t_{k}\right) \leq x\left(t_{k}\right) \leq \beta\left(t_{k}\right), \\
& \quad k=1,2, \ldots, m .
\end{aligned}
$$

Lemma 2 (see [5]). Assume that $s \in[0, T), x \in P C^{1}(J), p, q \in$ $P C(J), a_{i} \geq 0, b_{i}, i=1,2, \ldots, m$, are constants such that

$$
\begin{gathered}
x^{\prime}(t) \leq p(t) x(t)+q(t), \quad t \in[s, T), t \neq t_{k}, \\
x\left(t_{k}^{+}\right) \leq a_{k} x\left(t_{k}\right)+b_{k}, \quad t_{k} \in[s, T), k=1,2, \ldots, m ;
\end{gathered}
$$


then

$$
\begin{aligned}
& x(t) \leq x\left(s^{+}\right)\left(\prod_{s<t_{k}<t} a_{k}\right) \exp \left(\int_{s}^{t} p(u) d u\right) \\
&+\sum_{s<t_{k}<t}\left(\prod_{t_{k}<t_{i}<t} a_{i}\right) \exp \left(\int_{t_{k}}^{t} p(\tau) d \tau\right) b_{k} \\
&+\int_{s}^{t}\left(\prod_{u<t_{k}<t} a_{k}\right) \exp \left(\int_{u}^{t} p(\tau) d \tau\right) q(u) d u \\
& t \in[s, T] .
\end{aligned}
$$

Lemma 3. Assume that $x \in P C^{1}(J), M_{1}>0, M_{i} \geq 0, i=$ $2,3,4$, and $0 \leq L_{k}<1,(k=1,2, \ldots, m)$ such that

$$
\begin{aligned}
x^{\prime}(t) \leq & -M_{1} x(t)-M_{2} x(\theta(t))-M_{3}(K x)(t) \\
& -M_{4}(H x)(t)-a_{x}(t), \quad t \in J, t \neq t_{k}, \\
\Delta x\left(t_{k}\right) \leq & -L_{k} x\left(t_{k}\right)-L_{k} a_{x k}, \quad k=1,2, \ldots, m,
\end{aligned}
$$

where

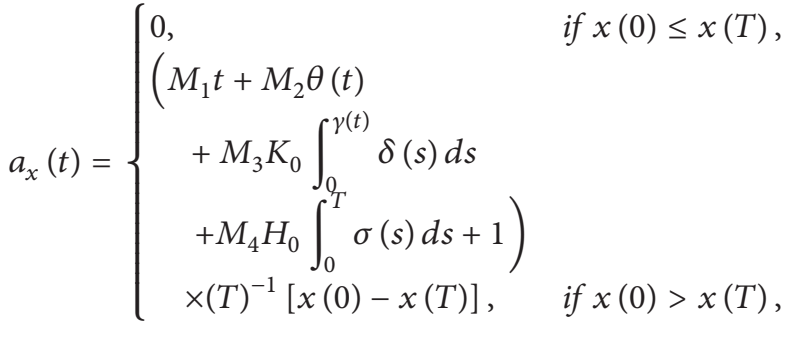

$$
\begin{aligned}
& a_{x k}= \begin{cases}0, & \text { if } x(0) \leq x(T), \\
\frac{t_{k}}{T}[x(0)-x(T)], & \text { if } x(0)>x(T) .\end{cases}
\end{aligned}
$$

If

$$
\begin{gathered}
M_{2} \int_{0}^{T}\left(\prod_{t<t_{k}<T}\left(1-L_{k}\right)\right) e^{M_{1}(t-\theta(t))} d t \\
+M_{3} \int_{0}^{T}\left(\int_{0}^{\gamma(t)}\left(\prod_{t<t_{k}<T}\left(1-L_{k}\right)\right)\right. \\
\left.\times M_{4}^{M_{1}(t-\delta(s))} k(t, s) d s\right) d t \\
\int_{0}^{T}\left(\int_{0}^{T}\left(\prod_{t<t_{k}<T}\left(1-L_{k}\right)\right)\right. \\
\leq e^{-M_{1} T} \prod_{j=1}^{m}\left(1-L_{k}\right)^{2},
\end{gathered}
$$

then $x(t) \leq 0$ on $J$.
Proof.

Case $1(x(0) \leq x(T))$. Let $v(t)=x(t) e^{M_{1} t}$; then $v \in P C^{1}(J)$ and

$$
\begin{gathered}
v^{\prime}(t) \leq-M_{2} e^{M_{1}(t-\theta(t))} v(\theta(t))-M_{3}\left(K_{1} v\right)(t) \\
-M_{4}\left(H_{1} v\right)(t), \quad t \in J^{\prime}, \\
\Delta v\left(t_{k}\right) \leq-L_{k} v\left(t_{k}\right), \quad k=1,2, \ldots, m, \\
v(0) \leq v(T) e^{-M_{1} T},
\end{gathered}
$$

where

$$
\begin{gathered}
\left(K_{1} v\right)(t)=\int_{0}^{\gamma(t)} k_{1}(t, s) v(\delta(s)) d s, \\
\left(H_{1} v\right)(t)=\int_{0}^{T} h_{1}(t, s) v(\sigma(s)) d s, \\
k_{1}(t, s)=e^{M_{1}(t-\delta(s))} k(t, s), \\
h_{1}(t, s)=e^{M_{1}(t-\sigma(s))} h(t, s) .
\end{gathered}
$$

Obviously, $v(t) \leq 0$ implies that $x(t) \leq 0$. To show $v(t) \leq 0$, suppose, on the contrary, that $v(\widetilde{t})>0$ for some $\tilde{t} \in J$. Then there are two possible cases:

(a) $v(t) \geq 0$ for all $t \in J$;

(b) there exists $t_{1}^{*} \in J$ such that $v\left(t_{1}^{*}\right)<0$.

In case (a), (17) implies that $v^{\prime}(t) \leq 0$ for $t \in J^{\prime}$ and $\Delta v\left(t_{k}\right) \leq 0(k=1,2, \ldots, m)$; hence, $v(t)$ is nonincreasing in $J$; then $v(0) \geq v(\widetilde{t})>0$ and $v(0) \geq v(T)$. On the other hand, $v(0)=x(0) \leq x(T)<x(T) e^{M_{1} T}=v(T)$, which is a contradiction.

In case (b), let $t^{*}=\max \left\{t \in J, \inf _{s \in J} v(s)=v(t)=-\lambda, \lambda>\right.$ $0\}$. We only consider $t^{*} \in\left(t_{p-1}, t_{p}\right]$ for some $p \in\{1,2, \ldots, m+$ $1\}$, as the proof is similar for the case $t^{*}=t_{p-1}^{+}$. Next, we consider two subcases.

Subcase $I(v(T) \geq 0)$. From (17), we get

$$
\begin{aligned}
v^{\prime}(t) \leq & \lambda M_{2} e^{M_{1}(t-\theta(t))}+\lambda M_{3} \int_{0}^{\gamma(t)} k_{1}(t, s) d s \\
& +\lambda M_{4} \int_{0}^{T} h_{1}(t, s) d s .
\end{aligned}
$$


From Lemma (14), we have

$$
\begin{aligned}
v(T) \leq & v\left(t^{*}\right) \prod_{t^{*}<t_{k}<T}\left(1-L_{k}\right) \\
& +\lambda M_{2} \int_{t^{*}}^{T}\left(\prod_{t<t_{k}<T}\left(1-L_{k}\right)\right) e^{M_{1}(t-\theta(t))} d t \\
& +\lambda M_{3} \int_{t^{*}}^{T}\left(\int_{0}^{\gamma(t)}\left(\prod_{t<t_{k}<T}\left(1-L_{k}\right)\right)\right. \\
& +\lambda M_{4} \int_{t^{*}}^{T}\left(\int_{0}^{T}\left(\prod_{t<t_{k}<T}\left(1-L_{k}\right)\right)\right. \\
& +\lambda M_{4} \int_{t^{*}}^{T}\left(\int_{0}^{M_{1}(t-\delta(s))} k(t, s) d s\right) d t \\
& +\lambda M_{3} \int_{t^{*}}^{T}\left(\prod_{t<t_{k}<T}\left(1-L_{k}\right)\right) \\
& +\lambda M_{2} \int_{t^{*}}^{T}\left(\prod_{t<t_{k}<T}\left(1-e_{t<t_{k}<T}\left(1-L_{k}\right)\right)\right. \\
\leq & -\lambda \prod_{k=p}^{m}\left(1-L_{k}(t-\sigma(s)) h(t, s) d s\right) d t \\
& \left.\times e^{M_{1}(t-\sigma(s))} h(t, s) d s\right) d t
\end{aligned}
$$

hence,

$$
\begin{aligned}
v(T)< & -\lambda\left(\prod_{k=1}^{m}\left(1-L_{k}\right)\right) \\
& +\lambda M_{2} \int_{0}^{T}\left(\prod_{t<t_{k}<T}\left(1-L_{k}\right)\right) e^{M_{1}(t-\theta(t))} d t \\
& +\lambda M_{3} \int_{0}^{T}\left(\int_{0}^{\gamma(t)}\left(\prod_{t<t_{k}<T}\left(1-L_{k}\right)\right)\right. \\
& \left.\times e^{M_{1}(t-\delta(s))} k(t, s) d s\right) d t
\end{aligned}
$$

$$
\begin{aligned}
+\lambda M_{4} \int_{0}^{T}\left(\int_{0}^{T}\left(\prod_{t<t_{k}<T}\left(1-L_{k}\right)\right)\right. \\
\left.\times e^{M_{1}(t-\sigma(s))} h(t, s) d s\right) d t .
\end{aligned}
$$

This yields

$$
\begin{gathered}
\prod_{k=1}^{m}\left(1-L_{k}\right)<M_{2} \int_{0}^{T}\left(\prod_{t<t_{k}<T}\left(1-L_{k}\right)\right) e^{M_{1}(t-\theta(t))} d t \\
+M_{3} \int_{0}^{T}\left(\int_{0}^{\gamma(t)}\left(\prod_{t<t_{k}<T}\left(1-L_{k}\right)\right)\right. \\
\times M_{4} \int_{0}^{T}\left(\int_{0}^{T}\left(\prod_{t<t_{k}<T}\left(1-L_{k}\right)\right)\right. \\
\left.\times e^{M_{1}(t-\delta(s))} k(t, s) d s\right) d t \\
\end{gathered}
$$

It contradicts (16).

Subcase II $(v(T)<0)$. If $t^{*}<\tilde{t}$, similar to the subcase I, it also yields a contradiction. We assume without loss of generality that $t^{*}>\tilde{t}$ and $\tilde{t} \in\left(t_{q}, t_{q+1}\right]$ for some $q \in\{0,1,2, \ldots, m-1\}$. By Lemma 2, we have

$$
\begin{aligned}
& v(\tilde{t}) \leq v(0) \prod_{0<t_{k}<\tilde{t}}\left(1-L_{k}\right) \\
&+\lambda M_{2} \int_{0}^{\tilde{t}}\left(\prod_{t<t_{k}<\tilde{t}}\left(1-L_{k}\right)\right) e^{M_{1}(t-\theta(t))} d t \\
&+\lambda M_{3} \int_{0}^{\tilde{t}}\left(\int_{0}^{\gamma(t)}\left(\prod_{t<t_{k}<\tilde{t}}\left(1-L_{k}\right)\right)\right. \\
&+\lambda M_{4} \int_{0}^{\tilde{t}}\left(\int_{0}^{T}\left(\prod_{t<t_{k}<\tilde{t}}\left(1-L_{k}\right)\right)\right. \\
&\left.\times e^{M_{1}(t-\delta(s))} k(t, s) d s\right) d t
\end{aligned}
$$


Noting that $v(0) \leq v(T) e^{-M_{1} T}$, we get

$$
\begin{aligned}
0<v(\tilde{t}) \leq & v(T) e^{-M_{1} T} \prod_{K=1}^{q}\left(1-L_{k}\right) \\
& +\lambda M_{2} \int_{0}^{\tilde{t}}\left(\prod_{t<t_{k}<\tilde{t}}\left(1-L_{k}\right)\right) e^{M_{1}(t-\theta(t))} d t \\
& +\lambda M_{3} \int_{0}^{\tilde{t}}\left(\int_{0}^{\gamma(t)}\left(\prod_{t<t_{k}<\tilde{t}}\left(1-L_{k}\right)\right)\right. \\
& \left.\times e^{M_{1}(t-\delta(s))} k(t, s) d s\right) d t \\
& +\lambda M_{4} \int_{0}^{\tilde{t}}\left(\int_{0}^{T}\left(\prod_{t<t_{k}<\tilde{t}}\left(1-L_{k}\right)\right)\right. \\
& \left.\times e^{M_{1}(t-\sigma(s))} h(t, s) d s\right) d t .
\end{aligned}
$$

Hence,

$$
\begin{gathered}
v(T) \prod_{K=1}^{q}\left(1-L_{k}\right) \\
\geq-\lambda M_{2} e^{M_{1} T} \int_{0}^{\tilde{t}}\left(\prod_{t<t_{k}<\tilde{t}}\left(1-L_{k}\right)\right) e^{M_{1}(t-\theta(t))} d t \\
-\lambda M_{3} e^{M_{1} T} \int_{0}^{\tilde{t}}\left(\int_{0}^{\gamma(t)}\left(\prod_{t<t_{k}<\tilde{t}}\left(1-L_{k}\right)\right)\right. \\
\quad-\lambda M_{4} e^{M_{1} T} \int_{0}^{\tilde{t}}\left(\int_{0}^{T}\left(\prod_{t<t_{k}<\tilde{t}}\left(1-L_{k}\right)\right)\right. \\
\left.\times e^{M_{1}(t-\delta(s))} k(t, s) d s\right) d t
\end{gathered}
$$

From (20) and (25), we obtain

$$
\begin{aligned}
\prod_{k=p}^{m}\left(1-L_{k}\right)\left(\prod_{j=1}^{q}\left(1-L_{k}\right)\right) \\
\leq M_{2} e^{M_{1} T} \int_{0}^{\tilde{t}}\left(\prod_{t<t_{k}<\tilde{t}}\left(1-L_{k}\right)\right) e^{M_{1}(t-\theta(t))} d t \\
+M_{3} e^{M_{1} T} \int_{0}^{\tilde{t}}\left(\int_{0}^{\gamma(t)}\left(\prod_{t<t_{k}<\tilde{t}}\left(1-L_{k}\right)\right)\right.
\end{aligned}
$$

$$
\begin{gathered}
+M_{4} e^{M_{1} T} \int_{0}^{\tilde{t}}\left(\int_{0}^{T}\left(\prod_{t<t_{k}<\tilde{t}}\left(1-L_{k}\right)\right)\right. \\
\left.\times e^{M_{1}(t-\sigma(s))} h(t, s) d s\right) d t \\
+M_{2} \prod_{j=1}^{q}\left(1-L_{k}\right) \int_{t^{*}}^{T}\left(\prod_{t<t_{k}<T}\left(1-L_{k}\right)\right) e^{M_{1}(t-\theta(t))} d t \\
+M_{3} \prod_{j=1}^{q}\left(1-L_{k}\right) \int_{t^{*}}^{T}\left(\int_{0}^{\gamma(t)}\left(\prod_{t<t_{k}<T}\left(1-L_{k}\right)\right)\right. \\
\times M_{4} \prod_{j=1}^{q}\left(1-L_{k}\right) \int_{t^{*}}^{T}\left(\int_{0}^{T}\left(\prod_{t<t_{k}<T}\left(1-L_{k}\right)\right)\right. \\
\left.\times e^{M_{1}(t-\delta(s))} k(t, s) d s\right) d t
\end{gathered}
$$

By multiplying $\prod_{j=q+1}^{m}\left(1-L_{k}\right)$ on both sides of (26), we have

$$
\begin{aligned}
\prod_{k=p}^{m}\left(1-L_{k}\right)\left(\prod_{j=1}^{m}\left(1-L_{k}\right)\right) \\
\leq M_{2} e^{M_{1} T} \int_{0}^{\tilde{t}}\left(\prod_{t<t_{k}<T}\left(1-L_{k}\right)\right) e^{M_{1}(t-\theta(t))} d t \\
+M_{3} e^{M_{1} T} \int_{0}^{\tilde{t}}\left(\int_{0}^{\gamma(t)}\left(\prod_{t<t_{k}<T}\left(1-L_{k}\right)\right)\right. \\
+M_{4} e^{M_{1} T} \int_{0}^{\tilde{t}}\left(\int_{0}^{T}\left(\prod_{t<t_{k}<T}\left(1-L_{k}\right)\right)\right. \\
+M_{3} \prod_{j=1}^{m}\left(1-L_{k}\right) \int_{t^{*}}^{T}\left(\int_{0}^{\gamma(t)}\left(\prod_{t<t_{k}<T}\left(1-L_{k}\right)\right) d t\right. \\
+M_{2} \prod_{j=1}^{m}\left(1-L_{k}\right) \int_{t^{*}}^{T}\left(\prod_{t<t_{k}<T}(1-s) d s\right) d t \\
\left.\quad \times e^{M_{1}(t-\sigma(s))} h(t, s) d s\right) d t \\
\end{aligned}
$$




$$
\begin{array}{r}
+M_{4} \prod_{j=1}^{m}\left(1-L_{k}\right) \int_{t^{*}}^{T}\left(\int_{0}^{T}\left(\prod_{t<t_{k}<T}\left(1-L_{k}\right)\right)\right. \\
\left.\times e^{M_{1}(t-\sigma(s))} h(t, s) d s\right) d t .
\end{array}
$$

Noting that $\prod_{j=1}^{m}\left(1-L_{k}\right)<1, e^{M_{1} T}>1$, we get

$$
\begin{aligned}
& \prod_{k=p}^{m}\left(1-L_{k}\right)\left(\prod_{j=1}^{m}\left(1-L_{k}\right)\right) \\
&<M_{2} e^{M_{1} T} \int_{0}^{T}\left(\prod_{t<t_{k}<T}\left(1-L_{k}\right)\right) e^{M_{1}(t-\theta(t))} d t \\
&+M_{3} e^{M_{1} T} \int_{0}^{T}\left(\int_{0}^{\gamma(t)}\left(\prod_{t<t_{k}<T}\left(1-L_{k}\right)\right)\right. \\
& \times M_{4} e^{M_{1} T} \int_{0}^{T}\left(\int_{0}^{T}\left(\prod_{t<t_{k}<T}\left(1-L_{k}\right)\right)\right. \\
&\left.\times e^{M_{1}(t-\delta(s))} k(t, s) d s\right) d t
\end{aligned}
$$

This implies

$$
\begin{aligned}
& e^{-M_{1} T} \prod_{j=1}^{m}\left(1-L_{k}\right)^{2} \\
& <M_{2} \int_{0}^{T}\left(\prod_{t<t_{k}<T}\left(1-L_{k}\right)\right) e^{M_{1}(t-\theta(t))} d t \\
& +M_{3} \int_{0}^{T}\left(\int_{0}^{\gamma(t)}\left(\prod_{t<t_{\mathrm{k}}<T}\left(1-L_{k}\right)\right)\right. \\
& +M_{4} \int_{0}^{T}\left(\int_{0}^{T}\left(\prod_{t<t_{k}<T}\left(1-L_{k}\right)\right)\right. \\
& \left.\times e^{M_{1}(t-\delta(s))} k(t, s) d s\right) d t
\end{aligned}
$$

which contradicts (16). This completes the proof for the case $x(0) \leq x(T)$.

Case $2(x(0)>x(T))$. Let $v(t)=x(t)+(t / T)[x(0)-x(T)]$; then $v(0)=x(0)=v(T)$, and

$$
\begin{aligned}
& v^{\prime}(t)+ M_{1} v(t)+M_{2} v(\theta(t)) \\
&+M_{3}(K v)(t)+M_{4}(H v)(t) \\
& \leq x^{\prime}(t)+M_{1} x(t)+M_{2} x(\theta(t)) \\
&+M_{3}(K x)(t)+M_{4}(H x)(t) \\
&+\left(M_{1} t+M_{2} \theta(t)+M_{3} K_{0} \int_{0}^{\gamma(t)} \delta(s) d s\right. \\
&\left.+M_{4} H_{0} \int_{0}^{T} \sigma(s) d s+1\right) \times(T)^{-1} \\
& \quad {[x(0)-x(T)] \leq 0, \quad t \neq t_{k}, t \in J, } \\
& \Delta v\left(t_{k}\right)=\Delta x\left(t_{k}\right) \leq-L_{k} x\left(t_{k}\right)-L_{k} \frac{t_{k}}{T}[x(0)-x(T)] \\
&=-L_{k} v\left(t_{k}\right), \quad k=1,2, \ldots, m .
\end{aligned}
$$

In view of Case 1 , we have $v(t) \leq 0$, for $t \in J$. Therefore, $x(t) \leq 0$ for $t \in J$. The proof of Lemma 3 is complete.

Lemma 4. Assume that $x \in P C^{1}(J), M_{1}>0, M_{i} \geq 0, i=$ $2,3,4, \eta \in P C(J)$, and $0 \leq L_{k}<1, k=1,2, \ldots, m$. If

$$
\left(M_{2} T+M_{3} T^{2} K_{0}+M_{4} T^{2} H_{0}+\sum_{k=1}^{m} L_{k}\right) \frac{e^{M_{1} T}}{e^{M_{1} T}-1}<1
$$

then the impulsive differential problem

$$
\begin{gathered}
x^{\prime}(t)=-M_{1} x(t)-M_{2} x(\theta(t))-M_{3}(K x)(t) \\
-M_{4}(H x)(t)+\eta(t), \quad t \in J, t \neq t_{k}, \\
\Delta x\left(t_{k}\right)=-L_{k} x\left(t_{k}\right)+d_{k}, \quad d_{k} \in R, k=1,2, \ldots, m, \\
x(0)=x(T),
\end{gathered}
$$

has a unique solution.

Proof. Define a map $A: P C(J) \rightarrow P C(J)$ by

$$
\begin{aligned}
{[A x](t)=} & \int_{0}^{T} G(t, s)\left(\eta(s)-M_{2} x(\theta(s))\right. \\
& \left.-M_{3}(K x)(s)-M_{4}(H x)(s)\right) d s \\
& +\sum_{k=1}^{m} G\left(t, t_{k}\right)\left(-L_{k} x\left(t_{k}\right)+d_{k}\right),
\end{aligned}
$$


where

$$
G(t, s)= \begin{cases}\frac{e^{-M_{1}(t-s)}}{1-e^{-M_{1} T}}, & 0 \leq s<t \leq T, \\ \frac{e^{-M_{1}(T+t-s)}}{1-e^{-M_{1} T}}, & 0 \leq t \leq s \leq T .\end{cases}
$$

It is easy to verify that $x$ is a solution of (32) if and only if $x$ is a fixed point of $A$. For any $x, y \in P C(J)$, we have

$$
\begin{aligned}
\mid[A x]( & t)-[A y](t) \mid \\
\leq & \left|M_{2} \int_{0}^{T} G(t, s)(y(\theta(s))-x(\theta(s))) d s\right| \\
& +\mid M_{3} \int_{0}^{T} \int_{0}^{\gamma(s)} G(t, s) k(s, u) \\
& +\mid M_{4} \iint_{0}^{T} G(t, s) h(s, u) \\
& \times(y(\delta(u))-x(\delta(u))) d u d s \mid \\
& +\left|\sum_{k=1}^{m} G\left(t, t_{k}\right) L_{k}\left(y\left(t_{k}\right)-x\left(t_{k}\right)\right)\right| \\
\leq & \left(M_{2} T+M_{3} T^{2} K_{0}+M_{4} T^{2} H_{0}+\sum_{k=1}^{m} L_{k}\right) \\
& \times \frac{e^{M_{1} T}}{e^{M_{1} T}-1}\|x-y\| ; \\
&
\end{aligned}
$$

this implies

$$
\begin{aligned}
\|[A x] & -[A y] \| \\
\leq & \left(M_{2} T+M_{3} T^{2} K_{0}+M_{4} T^{2} H_{0}+\sum_{k=1}^{m} L_{k}\right) \\
& \times \frac{e^{M_{1} T}}{e^{M_{1} T}-1}\|x-y\| .
\end{aligned}
$$

Condition (31) implies that $A$ is a contraction mapping. Banach's fixed point theorem implies that $A$ has a unique fixed point, and so (32) has a unique solution. The proof is complete.

\section{Main Results}

Theorem 5. Assume that $\alpha$ and $\beta$ are the lower solution and upper solution for (1) with $\alpha \leq \beta$ on $J$, respectively. Further, suppose that $\left(H_{1}\right),\left(H_{2}\right),(16)$, and (31) are satisfied. Then, (1) possesses in $[\alpha, \beta]$ the minimal and maximal solution $u, v \in P C^{1}(J)$, respectively. Moreover, there exist the monotone sequences $\left\{u_{n}\right\} \nearrow u$ and $\left\{v_{n}\right\} \searrow v$ uniformly on $J$, respectively.
Proof. For all $\psi \in[\alpha, \beta]$, we consider linear impulsive differential equation

$$
\begin{gathered}
y^{\prime}(t)=-M_{1} y(t)-M_{2} y(\theta(t))-M_{3}(K y)(t) \\
-M_{4}(H y)(t)+\eta(t), \quad t \neq t_{k}, \quad t \in J, \\
\Delta y\left(t_{k}\right)=I_{k}\left(\psi\left(t_{k}\right)\right)-L_{k}\left(y\left(t_{k}\right)-\psi\left(t_{k}\right)\right), \\
k=1,2, \ldots, m, \\
y(0)=y(T),
\end{gathered}
$$

where

$$
\begin{aligned}
\eta(t)= & f(t, \psi(t), \psi(\theta(t)),(K \psi)(t),(H \psi)(t))+M_{1} \psi(t) \\
& +M_{2} \psi(\theta(t))+M_{3}(K \psi)(t)+M_{4}(H \psi)(t) .
\end{aligned}
$$

By Lemma 4, one can see that (37) has a unique solution $y \in$ $P C^{1}(J)$. Denote that $A \psi$ is a unique solution of (37).

Let $u_{0}=\alpha, v_{0}=\beta$ and $u_{n}=A u_{n-1}, v_{n}=A v_{n-1}$. We will show that

(a) $u_{0} \leq A u_{0}, A v_{0} \leq v_{0}$,

(b) $A$ is nondecreasing in $\left[u_{0}, v_{0}\right]$.

The Proof of Property (a). Let $p=u_{0}-u_{1}$; since $u_{1}(0)=$ $u_{1}(T)$, it follows that $p(0)-p(T)=u_{0}(0)-u_{0}(T)$, and

$$
\begin{aligned}
p^{\prime}(t)= & u_{0}^{\prime}(t)-u_{1}^{\prime}(t) \\
\leq & f\left(t, u_{0}(t), u_{0}(\theta(t)),\left(K u_{0}\right)(t),\left(H u_{0}\right)(t)\right) \\
& -a_{u_{0}}(t)+M_{1}\left(u_{1}(t)\right)+M_{2}\left(u_{1}(\theta(t))\right) \\
& +M_{3}\left(K u_{1}\right)(t)+M_{4}\left(H u_{1}\right)(t) \\
& -f\left(t, u_{0}(t), u_{0}(\theta(t)),\left(K u_{0}\right)(t),\left(H u_{0}\right)(t)\right) \\
& -M_{1} u_{0}(t)-M_{2} u_{0}(\theta(t)) \\
& -M_{3}\left(K u_{0}\right)(t)-M_{4}\left(H u_{0}\right)(t) \\
= & -M_{1} p(t)-M_{2} p(\theta(t))-M_{3}(K p)(t) \\
& -M_{4}(H p)(t)-a_{p}(t), \quad t \neq t_{k}, t \in J, \\
\Delta p\left(t_{k}\right)= & \Delta u_{0}\left(t_{k}\right)-\Delta u_{1}\left(t_{k}\right) \\
\leq & I_{k}\left(u_{0}\left(t_{k}\right)\right)-a_{u_{0} k}-I_{k}\left(u_{0}\left(t_{k}\right)\right) \\
& +L_{k}\left(u_{1}\left(t_{k}\right)-u_{0}\left(t_{k}\right)\right) \\
= & -L_{k} p\left(t_{k}\right)-a_{p k},
\end{aligned}
$$


where

$$
\begin{aligned}
& a_{u_{0}}(t)=a_{p}(t) \\
& = \begin{cases}0, & \text { if } p(0) \leq p(T), \\
& +M_{1} t+M_{2} K_{0} \int_{0}^{\gamma(t)} \delta(s) d s \\
+ & \left.M_{4} H_{0} \int_{0}^{T} \sigma(s) d s+1\right) \\
\times(T)^{-1}[p(0)-p(T)], & \text { if } p(0)>p(T),\end{cases} \\
& a_{u_{0} k}=a_{p k}= \begin{cases}0, & \text { if } p(0) \leq p(T), \\
\frac{t_{k}}{T}[p(0)-p(T)], & \text { if } p(0)>p(T) .\end{cases}
\end{aligned}
$$

By Lemma 3, we have $p(t) \leq 0$, for $t \in J$; that is, $u_{0} \leq A u_{0}$. Analogously, it is proved that $A v_{0} \leq v_{0}$.

Next, we prove the property (b). Let $\psi_{1}, \psi_{2} \in\left[u_{0}, v_{0}\right]$ with $\psi_{1} \leq \psi_{2}, \omega_{1}=A \psi_{1}, \omega_{2}=A \psi_{2}$, and $p=\omega_{1}-\omega_{2}$; then, from $\left(\mathrm{H}_{1}\right)$ and $\left(\mathrm{H}_{2}\right)$, we have that, for $t \neq t_{k}$,

$$
\begin{aligned}
p^{\prime}(t)= & \omega_{1}^{\prime}(t)-\omega_{2}^{\prime}(t)=-M_{1} \omega_{1}(t)-M_{2} \omega_{1}(\theta(t)) \\
& -M_{3} K\left(\omega_{1}\right)(t)-M_{4} H\left(\omega_{1}\right)(t) \\
& +f\left(t, \psi_{1}(t), \psi_{1}(\theta(t)),\left(K \psi_{1}\right)(t),\left(H \psi_{1}\right)(t)\right) \\
& +M_{1} \psi_{1}(t)+M_{2} \psi_{1}(\theta(t))+M_{3}\left(K \psi_{1}\right)(t) \\
& +M_{4}\left(H \psi_{1}\right)(t)+M_{1} \omega_{2}(t)+M_{2} \omega_{2}(\theta(t)) \\
& +M_{3} K\left(\omega_{2}\right)(t)+M_{4} H\left(\omega_{2}\right)(t) \\
& -f\left(t, \psi_{2}(t), \psi_{2}(\theta(t)),\left(K \psi_{2}\right)(t),\left(H \psi_{2}\right)(t)\right) \\
& -M_{1} \psi_{2}(t)-M_{2} \psi_{2}(\theta(t)) \\
& -M_{3}\left(K \psi_{2}\right)(t)-M_{4}\left(H \psi_{2}\right)(t) \\
\leq & M_{1}\left(\psi_{2}(t)-\psi_{1}(t)\right) \\
& +M_{2}\left(\psi_{2}(\theta(t))-\psi_{1}(\theta(t))\right) \\
& +M_{3}\left(\left(K \psi_{2}\right)(t)-\left(K \psi_{1}\right)(t)\right) \\
& +M_{4}\left(\left(H \psi_{2}\right)(t)-\left(H \psi_{1}\right)(t)\right)-M_{1} \omega_{1}(t) \\
& -M_{2} \omega_{1}(\theta(t))-M_{3} K\left(\omega_{1}\right)(t)-M_{4} H\left(\omega_{1}\right)(t) \\
& +M_{1} \psi_{1}(t)+M_{2} \psi_{1}(\theta(t))+M_{3}\left(K \psi_{1}\right)(t) \\
& +M_{4}\left(H \psi_{1}\right)(t)+M_{1} \omega_{2}(t)+M_{2} \omega_{2}(\theta(t)) \\
& +M_{3} K\left(\omega_{2}\right)(t)+M_{4} H\left(\omega_{2}\right)(t)-M_{1} \psi_{2}(t) \\
& -M_{2} \psi_{2}(\theta(t))-M_{3}\left(K \psi_{2}\right)(t)-M_{4}\left(H \psi_{2}\right)(t) \\
= & -M_{1} p(t)-M_{2} p(\theta(t)) \\
& -M_{3}(K p)(t)-M_{4}(H p)(t), \\
& =(t)
\end{aligned}
$$

and, for $t=t_{k}$,

$$
\begin{aligned}
\Delta p\left(t_{k}\right)= & \Delta \omega_{1}\left(t_{k}\right)-\Delta \omega_{2}\left(t_{k}\right) \\
= & I_{k}\left(\psi_{1}\left(t_{k}\right)\right)-L_{k}\left(\omega_{1}\left(t_{k}\right)-\psi_{1}\left(t_{k}\right)\right)-I_{k}\left(\psi_{2}\left(t_{k}\right)\right) \\
& +L_{k}\left(\omega_{2}\left(t_{k}\right)-\psi_{2}\left(t_{k}\right)\right) \\
\leq & L_{k}\left(\psi_{2}\left(t_{k}\right)-\psi_{1}\left(t_{k}\right)\right)-L_{k}\left(\omega_{1}\left(t_{k}\right)-\psi_{1}\left(t_{k}\right)\right) \\
& +L_{k}\left(\omega_{2}\left(t_{k}\right)-\psi_{2}\left(t_{k}\right)\right) \\
= & -L_{k} p\left(t_{k}\right) ;
\end{aligned}
$$

it is clear that $p(0)=p(T)$. It follows by Case 1 of Lemma 3 that $p(t) \leq 0$ for $t \in J$; that is, $A$ is monotonely nondecreasing in $\left[u_{0}, v_{0}\right]$.

It follows, from the properties (a) and (b), that

$$
\alpha \leq u_{1} \leq \cdots \leq u_{n} \leq v_{n} \cdots \leq v_{1} \leq \beta, \quad \text { on } J .
$$

By standard arguments, we conclude that there exist $u$ and $v$ such that

$$
\begin{gathered}
\lim _{n \rightarrow \infty} u_{n}=u(t), \quad \lim _{n \rightarrow \infty} v_{n}=v(t), \quad \text { uniformly on } J, \\
u_{n}(t) \leq u(t) \leq v(t) \leq v_{n}(t) .
\end{gathered}
$$

It is easy to show that $u$ and $v$ are solutions of (1) using $u_{n}, v_{n}$ satisfy the relations

$$
\begin{array}{rl}
u_{n+1}^{\prime}(t)= & -M_{1}\left(u_{n+1}(t)\right)-M_{2}\left(u_{n+1}(\theta(t))\right) \\
& -M_{3}\left(K u_{n+1}\right)(t)-M_{4}\left(H u_{n+1}\right)(t) \\
& +f\left(t, u_{n}(t), u_{n}(\theta(t)),\left(K u_{n}\right)(t),\left(H u_{n}\right)(t)\right) \\
& +M_{1} u_{n}(t)+M_{2} u_{n}(\theta(t)) \\
& +M_{3}\left(K u_{n}\right)(t)+M_{4}\left(H u_{n}\right)(t), \quad t \in J^{\prime}, \\
\Delta u_{n+1}\left(t_{k}\right)=I_{k}\left(u_{n}\left(t_{k}\right)\right)-L_{k}\left(u_{n+1}\left(t_{k}\right)-u_{n}\left(t_{k}\right)\right), & k=1,2, \ldots, m, \\
v_{n+1}^{\prime}(t)= & -M_{1}\left(v_{n+1}(t)\right)-M_{2}\left(v_{n+1}(\theta(t))\right) \\
& -M_{3}\left(K v_{n+1}\right)(t)-M_{4}\left(H v_{n+1}\right)(t) \\
& +f\left(t, v_{n}(t), v_{n}(\theta(t)),\left(K v_{n}\right)(t),\left(H v_{n}\right)(t)\right) \\
& +M_{1} v_{n}(t)+M_{2} v_{n}(\theta(t)) \\
& +M_{3}\left(K v_{n}\right)(t)+M_{4}\left(H v_{n}\right)(t), \quad t \in J^{\prime}, \\
\Delta v_{n+1} & \left.t_{k}\right)=I_{k}\left(v_{n}\left(t_{k}\right)\right)-L_{k}\left(v_{n+1}\left(t_{k}\right)-v_{n}\left(t_{k}\right)\right), \\
k & k 1,2, \ldots, m, \\
v_{n+1}(0)=v_{n+1}(T) .
\end{array}
$$


Taking the limit as $n \rightarrow \infty$ on both sides of above relations, we have

$$
\begin{gathered}
u^{\prime}(t)=f(t, u(t), u(\theta(t)),(K u)(t),(H u)(t)), \\
t \neq t_{k}, t \in J, \\
\Delta u\left(t_{k}\right)=I_{k}\left(u\left(t_{k}\right)\right), \quad k=1,2, \ldots, m, \\
u(0)=u(T), \\
v^{\prime}(t)=f(t, v(t), v(\theta(t)),(K v)(t),(H v)(t)), \\
t \neq t_{k}, t \in J, \\
\Delta v\left(t_{k}\right)=I_{k}\left(v\left(t_{k}\right)\right), \quad k=1,2, \ldots, m, \\
v(0)=v(T) .
\end{gathered}
$$

Equation (46) show that $u$ and $v$ are solutions of (1). Finally, we prove that, if $z \in[\alpha, \beta]$ is any solution of $(1)$, then $u(t) \leq z(t) \leq v(t)$ on $J$. To this end, we assume, without loss of generality, that $u_{n}(t) \leq z(t) \leq v_{n}(t)$ for some $n$, since $\alpha(t) \leq z(t) \leq \beta(t)$. From property (b), we can get

$$
u_{n+1}(t) \leq z(t) \leq v_{n+1}(t), \quad t \in J .
$$

Since $u_{0}(t)=\alpha(t) \leq z(t) \leq \beta(t)=v_{0}(t), t \in J$, by induction, we can conclude that

$$
u_{n}(t) \leq z(t) \leq v_{n}(t), \quad \forall n .
$$

Passing to the limit as $n \rightarrow \infty$, we obtain

$$
u(t) \leq z(t) \leq v(t), \quad t \in J .
$$

This ends the proof.

\section{Conflict of Interests}

The author declares that there is no conflict of interests regarding the publication of this paper.

\section{Acknowledgments}

This work is supported by the National Science Foundation (11171085) and Key Project of Hunan Province Education Department (no. 11A095), China.

\section{References}

[1] D. D. Baĭnov and P. S. Simeonov, Syetems with impulse Effect: Stability Theory and Applications, Chichester, UK, 1989.

[2] V. D. Mill'man and A. D. Myshkis, "On the stability of motion in the presence of impulsive," Siberian Mathematical Journal, vol. 1, pp. 233-237, 1960.

[3] A. M. Samoilenko and N. A. Perestyuk, Differential Equations with Impulsive Effect, Kiev State University, 1980, (Russian).

[4] V. Lakshmikantham, D. D. Baĭnov, and P. S. Simeonov, Theory of Impulsive Differential Equations, vol. 6, World Scientific, Singapore, 1989.
[5] D. D. Bainov and P. S. Simeonov, Impulsive Differential Equations: Periodic Solutions and Applications, Longman Scientific and Technical, Harlow, UK, 1993.

[6] J. Shen and J. Li, "Impulsive control for stability of Volterra functional differential equations," Journal for Analysis and its Applications, vol. 24, no. 4, pp. 721-734, 2005.

[7] I. Rachunkova and M. Tvrdy, "Existence results for impulsive second-order periodic problems," Nonlinear Analysis. Theory, Methods \& Applications A, vol. 59, no. 1-2, pp. 133-146, 2004.

[8] J. Shen, J. Li, and Q. Wang, "Boundedness and periodicity in impulsive ordinary and functional differential equations," Nonlinear Analysis. Theory, Methods \& Applications A, vol. 65, no. 10, pp. 1986-2002, 2006.

[9] J. L. Li, Boundary value problems and periodic solutions of impulsive differential equations [Ph.D. thesis], Hunan Normal University, 2006, Chinese.

[10] R. Liang and J. Shen, "Periodic boundary value problem for the first order impulsive functional differential equations," Journal of Computational and Applied Mathematics, vol. 202, no. 2, pp. 498-510, 2007.

[11] Y. Tian and W. Ge, "Applications of variational methods to boundary-value problem for impulsive differential equations," Proceedings of the Edinburgh Mathematical Society II, vol. 51, no. 2, pp. 509-527, 2008.

[12] J. Shen and J. Li, "Existence and global attractivity of positive periodic solutions for impulsive predator-prey model with dispersion and time delays," Nonlinear Analysis. Real World Applications, vol. 10, no. 1, pp. 227-243, 2009.

[13] J. J. Nieto and D. O'Regan, "Variational approach to impulsive differential equations," Nonlinear Analysis. Real World Applications, vol. 10, no. 2, pp. 680-690, 2009.

[14] Y. Shao and B. Dai, "The existence of exponential periodic attractor of impulsive BAM neural network with periodic coefficients and distributed delays," Neurocomputing, vol. 73, pp. 3123-3131, 2010.

[15] Z. Zhang and R. Yuan, "An application of variational methods to Dirichlet boundary value problem with impulses," Nonlinear Analysis. Real World Applications, vol. 11, no. 1, pp. 155-162, 2010.

[16] J. Zhou and Y. Li, "Existence of solutions for a class of secondorder Hamiltonian systems with impulsive effects," Nonlinear Analysis. Theory, Methods \& Applications A, vol. 72, no. 3-4, pp. 1594-1603, 2010.

[17] A. V. Arutyunov, D. Yu. Karamzin, and F. Pereira, "Pontryagin's maximum principle for constrained impulsive control problems," Nonlinear Analysis. Theory, Methods \& Applications A, vol. 75, no. 3, pp. 1045-1057, 2012.

[18] L. Nie, Z. Teng, and A. Torres, "Dynamic analysis of an SIR epidemic model with state dependent pulse vaccination," Nonlinear Analysis. Real World Applications, vol. 13, no. 4, pp. 1621-1629, 2012.

[19] G. S. Ladde, V. Lakshmikantham, and A. S. Vatsala, Monotone Iterative Techniques for Nonlinear Differential Equations, Pitman, London, UK, 1985.

[20] J. Li and J. Shen, "Periodic boundary value problems for impulsive differential-difference equations," Indian Journal of Pure and Applied Mathematics, vol. 35, no. 11, pp. 1265-1277, 2004.

[21] I. Rachunkova and M. Tvrdy, "Non-ordered lower and upper functions in second order impulsive periodic problems," Dynamics of Continuous, Discrete \& Impulsive Systems A, vol. 12, no. 3-4, pp. 397-415, 2005. 
[22] Z. He and J. Yu, "Periodic boundary value problem for firstorder impulsive functional differential equations," Journal of Computational and Applied Mathematics, vol. 138, no. 2, pp. 205-217, 2002.

[23] W. Ding, J. Mi, and M. Han, "Periodic boundary value problems for the first order impulsive functional differential equations," Applied Mathematics and Computation, vol. 165, no. 2, pp. 433446, 2005.

[24] X. Z. Liu and D. J. Guo, "Periodic boundary value problems for class of impulsive integrodifferential equations in Banach spaces," Journal of Mathematical Analysis and Applications, vol. 216, pp. 284-302, 1995.

[25] Z. He and X. He, "Monotone iterative technique for impulsive integro-differential equations with periodic boundary conditions," Computers \& Mathematics with Applications, vol. 48, no. 1-2, pp. 73-84, 2004.

[26] Z. He and X. He, "Periodic boundary value problems for first order impulsive integro-differential equations of mixed type," Journal of Mathematical Analysis and Applications, vol. 296, no. 1, pp. 8-20, 2004.

[27] J. J. Nieto and R. Rodríguez-López, "Existence and approximation of solutions for nonlinear functional differential equations with periodic boundary value conditions," Computers \& Mathematics with Applications, vol. 40, no. 4-5, pp. 433-442, 2000.

[28] J. J. Nieto and R. Rodríguez-López, "Remarks on periodic boundary value problems for functional differential equations," Journal of Computational and Applied Mathematics, vol. 158, no. 2, pp. 339-353, 2003. 


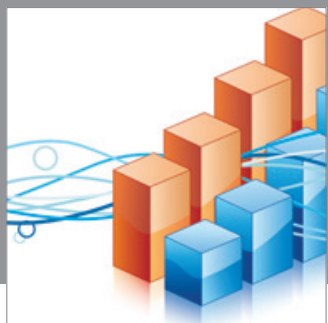

Advances in

Operations Research

mansans

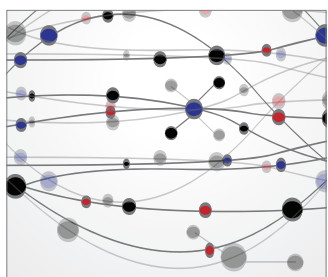

The Scientific World Journal
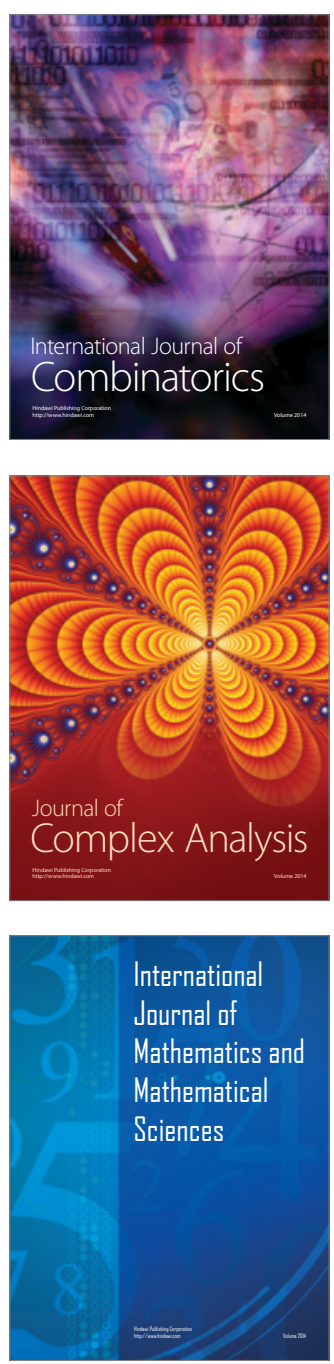
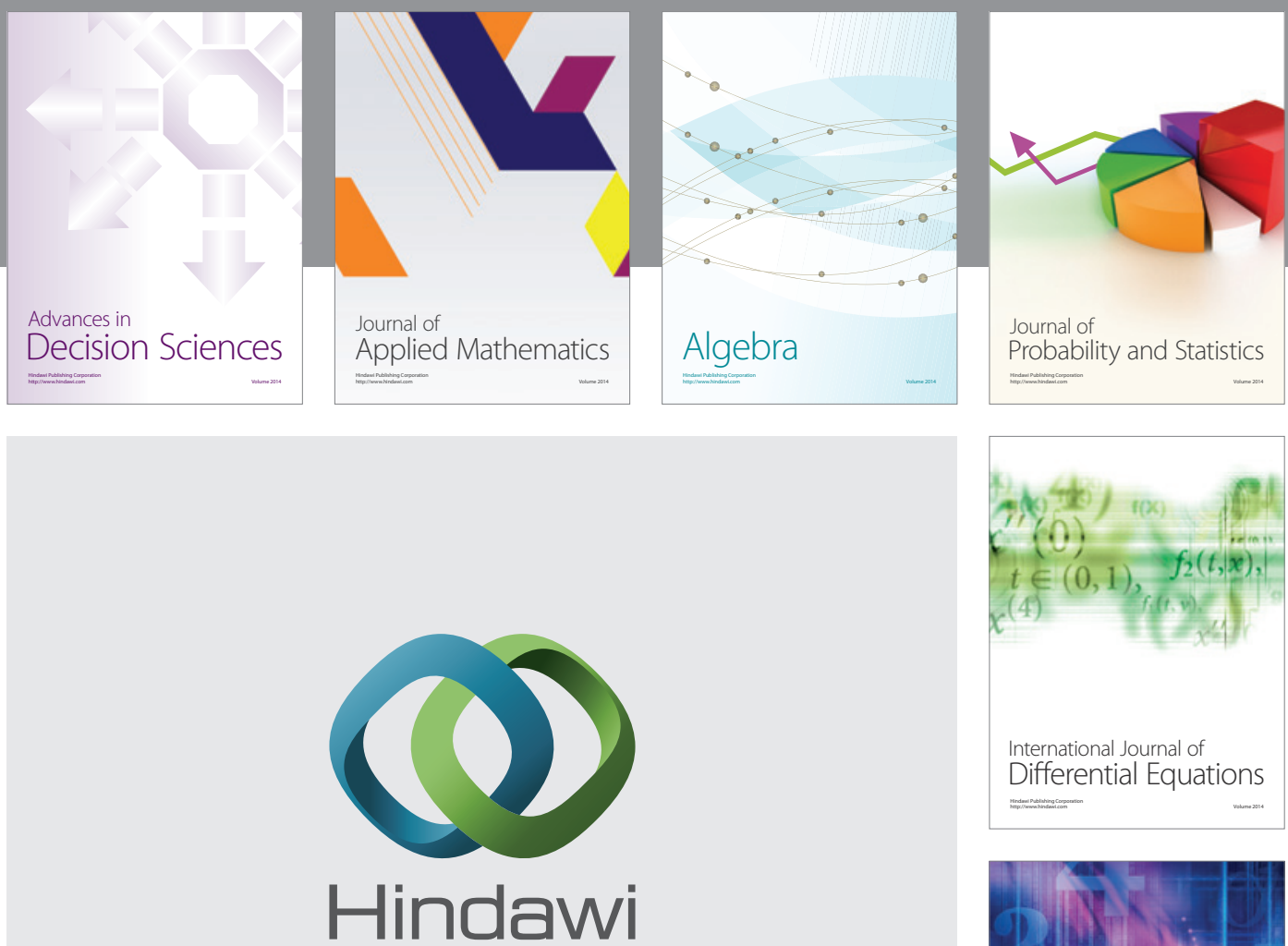

Submit your manuscripts at http://www.hindawi.com
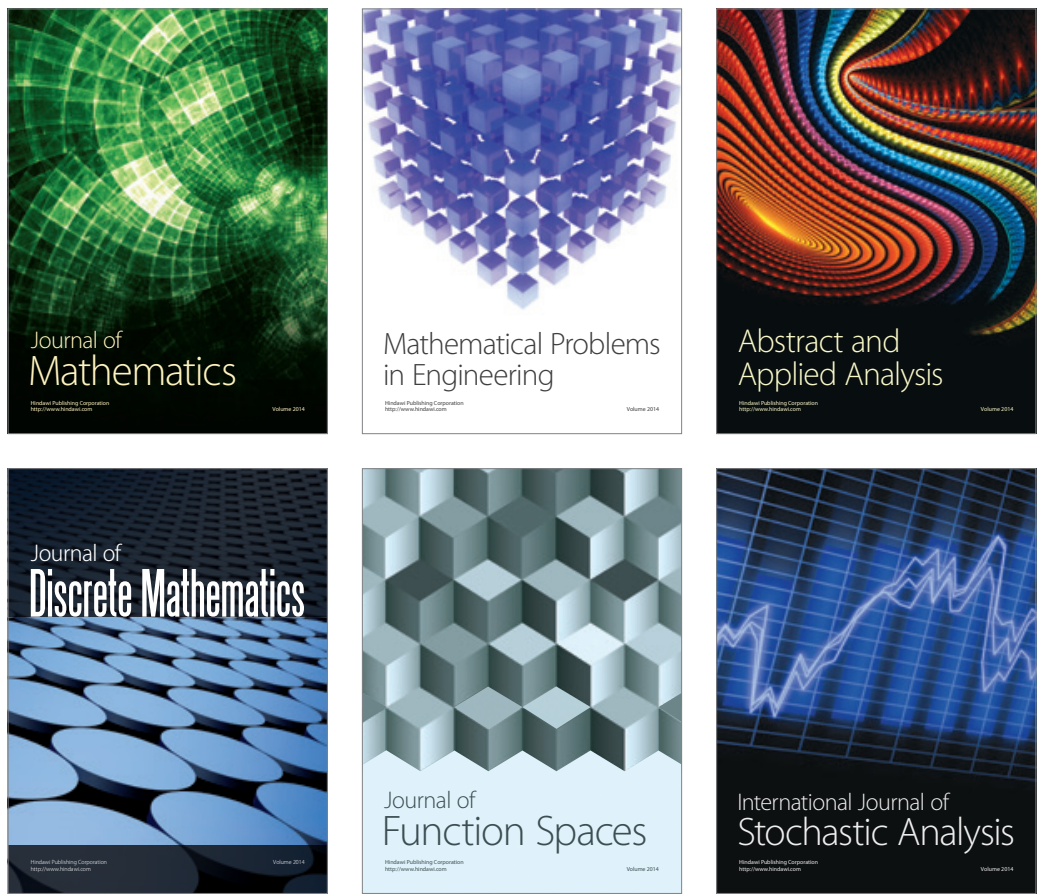

Journal of

Function Spaces

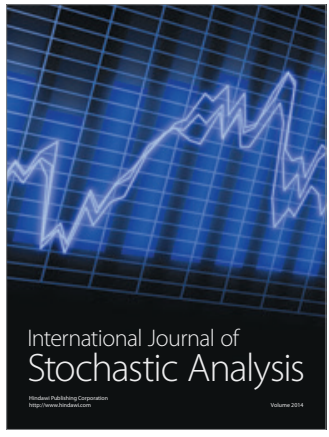

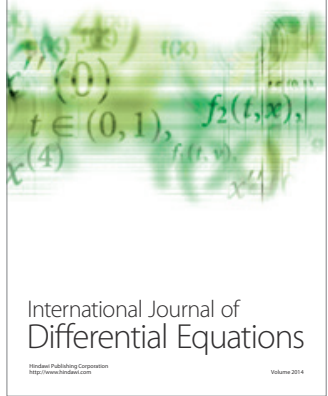
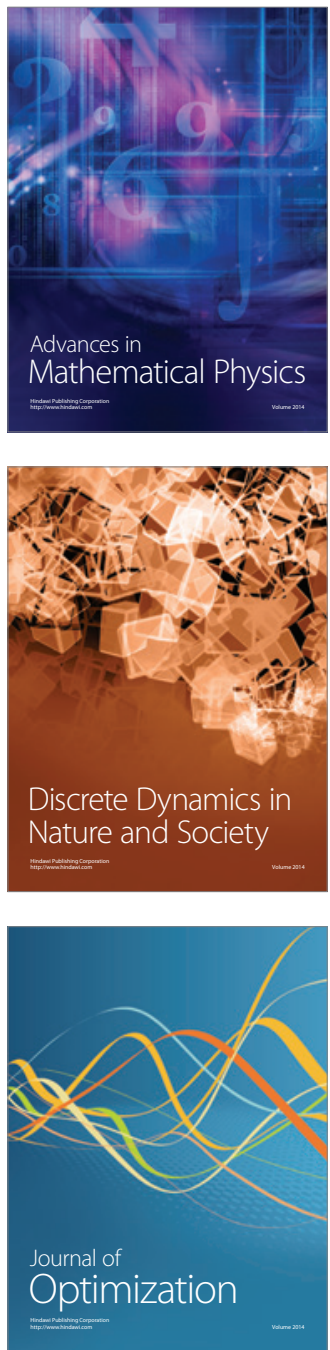